MATEC Web of Conferences 11,02006 (2014)

DOI: $10.1051 /$ matecconf / 20141102006

(C) Owned by the authors, published by EDP Sciences, 2014

\title{
Modeling and numerical simulation of static and dynamic behavior of multilayered plates with interface effects
}

\section{Modélisation et simulation numérique des comportements statique et dynamique des plaques multicouches à effets d'interfaces}

\author{
Smail ZAKI ${ }^{1}$, Lahcen AZRAR ${ }^{2,3}$ et Ahmed MOUCHTACHI ${ }^{1}$ \\ ${ }^{1}$ Equipe M2AI, Université Moulay Ismail, ENSAM, BP15290 El Mansour 50 000, Meknès-Maroc \\ ${ }^{2}$ Equipe de MMC, Département de Mathématiques, FST- Tanger, Université Abdelmalek Essaadi, BP416 Tanger-Maroc \\ ${ }^{3}$ Department of Mechanical Engineering, Faculty of Engineering, King Abdulaziz University, Jeddah, Saudi Arabia
}

\begin{abstract}
In Multilayered structures the interface effects have a wide range of applications in aerospace, automotive and especially in civil engineering. The design and construction of these structures and the account for interface effects require special expertise in modeling, simulation and implementation. Many studies in this case were conducted to address these issues. The objective of this work is the modeling and numerical simulation of static and dynamic behaviors of beams and plates multilayered structures with different types of interfaces. The focus was on the prediction of the behavior of stresses; shears and displacements depending on thickness. The interface can be elastic or viscoelastic of small or large thickness. The state space method has been developed for this purpose. Various types of rolled arbitrary number of isotropic or anisotropic layers structures were considered. The three-dimensional behavior is obtained for different types of static and dynamic loading. The results were compared with those based on the model of Stroh and on the various existing theories of beams and plates. The methodological approach, developed here, will be applied to thick structures, functionally graded, bimorph or multilayer structures and possibly piezoelectric or viscoelastic layered structures with interface effect
\end{abstract}

Résumé. Les structures multicouches à effets d'interface ont une large application en aéronautique, automobile et notamment en génie civil. La conception, la réalisation de ces structures ainsi que la prise en compte des effets d'interface nécessitent une compétence particulière en modélisation, simulation et mise en œuvre. De nombreuses études en l'occurrence ont été menées et ce pour répondre à ces problématiques. L'objectif de ce travail est la modélisation et simulation numérique des comportements statique et dynamique des structures poutres et plaques multicouches à différents types d'interfaces. L'accent a été mis sur la prédiction du comportement des contraintes, cisaillements et déplacements selon l'épaisseur. L'interface peut être élastique ou viscoélastique à petite ou à grande épaisseur. La méthode d'espace d'état a été élaborée pour cette fin. Différents types de structures laminées à nombre arbitraire de couches isotropes ou anisotropes ont été considérés. Le comportement tridimensionnel est obtenu pour différents types de chargement statiques et dynamiques. Les résultats obtenus ont été comparés avec ceux basés sur le modèle de Stroh ainsi que sur les différentes théories existantes des poutres et plaques. L'approche méthodologique, ainsi élaborée, va être appliquée aux structures épaisses, structures à comportement gradué, structures bilames ou multicouches et éventuellement à des structures à couches piézoélectriques ou viscoélastiques ou à effet d'interface.

\section{Introduction}

Il est particulièrement important de comprendre le comportement des structures avec des interfaces imparfaites pour les évaluer en intégralité au cours de 
leur durée de service. L'expérience a montré que de plus en plus dans ces interfaces il y a apparition des microfissures, des hétérogénéités, des dislocations ou des cavités relatives aux modes de fabrication. Aussi au cours du cycle de vie de ces structures, elles sont amenées à résister aux charges variées " fatigue », à un niveau de contrainte critique en statique, à des impacts et aussi exposées à des environnements très corrosifs. [1]

Généralement il est très difficile de prévoir le comportement exact de la liaison interlaminaire théoriquement ou expérimentalement. Certains modèles d'interface simplifiés ont été mis en place. Le plus populaire est le modèle linéaire de type ressort et son principe consiste à introduire une couche mince de matériau d'interphase entre deux couches adjacentes, qui devraient être représentatifs des adhésifs interlaminaires. Lorsque l'épaisseur de cette couche disparaît, la traction interfaciale deviennent continus, tandis que les déplacements dans l'autre côté de la couche d'interface deviennent discontinues. Un autre modèle de type dislocation a été introduit pour décrire mathématiquement l'effet d'une interface imparfaite sur le transfert de charge. Les conditions aux limites pour ce modèle sont similaires au modèle linéaire de type ressort, sauf que le saut en déplacement à l'interface est supposé être linéairement proportionnel au déplacement à l'interface du composant où la charge est appliquée [2].

Dans les travaux mentionnés ci-dessus, les réponses des stratifiés sous sollicitations statiques sont indépendants de la variable de temps. Notons que ces interfaces peuvent être visqueuses ou viscoélastiques et du coup leur comportement dynamique peut profondément influencer la totalité de la structure.

De nombreuses études en l'occurrence ont été menées et ce pour palier à cette problématique. He et Jiang [3] ont proposé une solution pour un stratifié avec interfaces visqueuse et ont montré que la réponse du stratifié varie clairement avec le temps, en particulier à l'étape initiale. Chen et Lee [4] ont proposé une méthode semi-analytique pour l'analyse des stratifiés avec différents angles d'orthotropie soumis à la flexion avec des interfaces visqueuses. Les deux études ont révélé que, lorsque le temps est grand, l'interface visqueuse perdrait la possibilité de transférer la contrainte de cisaillement parfaitement. Cependant, cela semble inadapté pour certains types de composites en pratiques et en particulier dans le cadre des petites déformations.

En fait, une interface viscoélastique sera plus appropriée pour caractériser le fluage et la relaxation qui sont les comportements les plus apparents dans des circonstances haute température [5]. Selon Fan et Wang [6], le comportement viscoélastique doit être envisagé lorsque la température de fonctionnement d'un solide est au-dessus de 1/3-1/2 de sa température de fusion (échelle de Kelvin). Il y a beaucoup de cas où l'interface doit être considérée comme viscoélastique. Yan et Chen [7] ont proposé une solution analytique pour une plaque à couches isotropes avec interfaces viscoélastiques soumises à la flexion cylindrique. La différence la plus importante par rapport aux interfaces visqueuses est que les viscoélastiques ne perdant pas la possibilité de transférer la contrainte de cisaillement avec le temps, ce qui semble plus réaliste dans certaines situations pratiques.

Toutefois, il convient de souligner que l'analyse exacte du l'élasticité 3D devient coûteuse en calcul lorsque le nombre de couches de stratifié augmente, en raison de l'implication d'un grand nombre de constantes d'intégration. [4]

L'objectif dans cet article est la modélisation et la simulation numérique des comportements statique et dynamique des plaques multicouches avec des interfaces viscoélastiques et subissant différents types de chargements dynamique ou statique et reposant sur différents types de fondation. L'accent est mis sur le comportement des contraintes, cisaillements et déplacements selon l'épaisseur pour des structures laminées ayant différents angles d'orthotropie.

\section{Formulation mathématique}

Considérons une plaque rectangulaire ayant une épaisseur $\mathrm{h}$, une longueur $l_{1}$ et une largeur $l_{2}$, et posée sur une fondation viscoélastique de type Winkler-Pasternak. « Figure 1 »

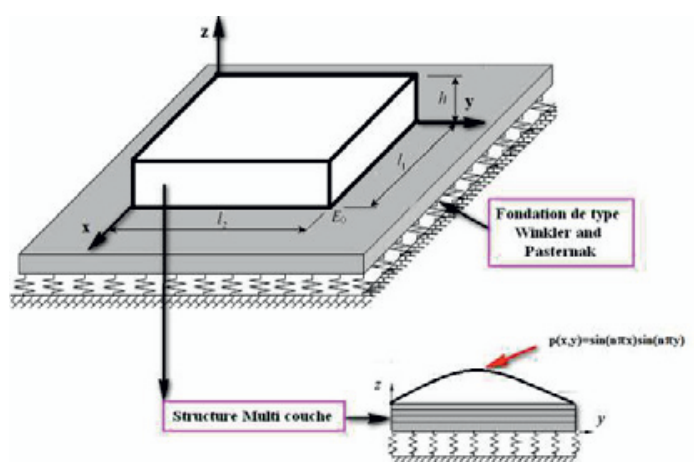

Fig 1. Géométrie du Problème

La plaque est considérée être multicouches et à interface. Chaque couche est constituée d'un matériau homogène, élastique et linéaire. Le comportement de cette plaque est gouverné par le système d'équation aux dérivées partielles suivant :

$$
\left\{\begin{array}{l}
\partial_{x} \sigma_{x}+\partial_{y} \tau_{x y}+\partial_{z} \tau_{x z}=\rho \frac{\partial^{2} u}{\partial t^{2}} \\
\partial_{x} \tau_{x y}+\partial_{y} \sigma_{y}+\partial_{z} \tau_{y z}=\rho \frac{\partial^{2} v}{\partial t^{2}} \\
\partial_{x} \tau_{x z}+\partial_{y} \tau_{y z}+\partial_{z} \sigma_{z}=\rho \frac{\partial^{2} w}{\partial t^{2}}
\end{array}\right.
$$


Où $\sigma_{x}, \sigma_{y}$, et $\sigma_{z}$ sont les composantes normales de contraintes; $\tau_{\mathrm{xy}}, \tau_{\mathrm{xz}}$ et $\tau_{\mathrm{yz}}$ sont les contraintes de cisaillement. $(\mathrm{u}, \mathrm{v})(\mathrm{x}, \mathrm{y}, \mathrm{z}, \mathrm{t})$ sont les déplacements dans le plan et $\mathrm{w}(\mathrm{x}, \mathrm{y}, \mathrm{z}, \mathrm{t})$ est le déplacement transversal.

En supposant que cette plaque est simplement supportée sur ces quatre cotés, les conditions de bords s'écrivent alors [8]:

$$
\left\{\begin{array}{lll}
w=v=\sigma_{x}=0 & \grave{a} & x=0, l_{1} \\
w=u=\sigma_{y}=0 & \grave{a} & y=0, l_{2}
\end{array}\right.
$$

On considère que chaque couche est constituée de matériau orthotrope dont la relation contrainte déformation est donnée pour chaque couche par :

$$
\left\{\begin{array}{l}
\sigma_{x} \\
\sigma_{y} \\
\sigma_{z} \\
\tau_{y z} \\
\tau_{x z} \\
\tau_{x y}
\end{array}\right\}=\left[\begin{array}{cccccc}
\hat{C}_{11} & \hat{C}_{12} & \hat{C}_{13} & 0 & 0 & \hat{C}_{16} \\
\hat{C}_{12} & \hat{C}_{22} & \hat{C}_{23} & 0 & 0 & \hat{C}_{26} \\
\hat{C}_{13} & \hat{C}_{23} & \hat{C}_{33} & 0 & 0 & \hat{C}_{36} \\
0 & 0 & 0 & \hat{C}_{44} & \hat{C}_{45} & 0 \\
0 & 0 & 0 & \hat{C}_{45} & \hat{C}_{55} & 0 \\
\hat{C}_{16} & \hat{C}_{26} & \hat{C}_{36} & 0 & 0 & \hat{C}_{66}
\end{array}\right]\left\{\begin{array}{l}
\varepsilon_{x} \\
\varepsilon_{y} \\
\varepsilon_{z} \\
\varepsilon_{y z} \\
\varepsilon_{x z} \\
\varepsilon_{x y}
\end{array}\right\}
$$

$\{\varepsilon\}$ est le tenseur de déformation et les coefficients $\hat{C}_{\mathrm{ij}}$ dépendent des caractéristiques du matériau et de l'angle d'orthotropie [8].

L'objectif de ce travail est l'élaboration d'une méthodologie mathématique et numérique pour la résolution du problème tridimensionnel (1).

\subsection{Méthode d'espace d'état}

On suppose que le mouvement est harmonique en temps et que la solution dans le plan (x, y) est connue et celle selon $\mathrm{z}$ est inconnue. On suppose que les contraintes et les déplacements de la plaque peuvent s'écrire sous forme de séries de Fourier comme suit:

$$
\left\{\begin{array}{l}
u \\
v \\
w \\
\sigma_{z} \\
\sigma_{x} \\
\sigma_{y} \\
\tau_{x y} \\
\tau_{x z} \\
\tau_{y z}
\end{array}\right\}=\sum_{n=0}^{\infty} \sum_{m=0}^{\infty}\left\{\begin{array}{l}
h U(\zeta, t) \cos (n \pi \xi) \sin (m \pi \eta) \\
h V(\zeta, t) \sin (n \pi \xi) \cos (m \pi \eta) \\
h W(\zeta, t) \sin (n \pi \xi) \sin (m \pi \eta) \\
\mu \sum_{\zeta}(\zeta, t) \sin (n \pi \xi) \sin (m \pi \eta) \\
\mu \sum_{\eta}(\zeta, t) \sin (n \pi \xi) \sin (m \pi \eta) \\
\mu \Gamma_{2}(\zeta, t) \cos (n \pi \xi) \cos (m \pi \eta) \\
\mu \Gamma_{1}(\zeta, t) \cos (n \pi \xi) \sin (m \pi \eta) \\
\mu \Gamma_{3}(\zeta, t) \sin (n \pi \xi) \cos (m \pi \eta)
\end{array}\right\}
$$

avec les coordonnées adimensionnelles $\xi=\mathrm{x} / \mathrm{a}, \eta=\mathrm{y} / \mathrm{b}$ et $\zeta=\mathrm{z} / \mathrm{h}$. h est l'épaisseur totale et $\mu$ est le coefficient de Lamé.

Sous l'hypothèse des petites déformations, le tenseur de déformation $\{\varepsilon\}$ est exprimé en fonction des déplacements (u, v, w). On insert les développements (4) dans les équations du mouvement (1) et on utilise les relations constitutives (3). En appliquant la transformée de Laplace et après des développements mathématiques, on se ramène au système différentiel du premier ordre suivant pour chaque couche :

$\frac{d}{d \zeta} \bar{\Psi}(\zeta, s)=A_{n m}(s) \bar{\Psi}(\zeta, s)$

avec $\quad \bar{\Psi}(\zeta, s)=\left(\begin{array}{llllll}\bar{\Sigma}_{\zeta} & \bar{U} & \bar{V} & \bar{W} & \bar{\Gamma}_{1} & \bar{\Gamma}_{3}\end{array}\right)$

Les coefficients de la matrice $\boldsymbol{A}_{\boldsymbol{n} m}$ sont donnés par:

$A_{n m}(s)=\left[\begin{array}{cccccc}0 & 0 & 0 & \frac{\rho s^{2} h^{2}}{\mu} & k n & k m \\ 0 & 0 & 0 & -k n & \frac{\mu}{\hat{C}_{55}} & 0 \\ 0 & 0 & 0 & -k m & 0 & \frac{\mu}{\hat{C}_{44}} \\ \frac{\mu}{h \hat{C}_{33}} & \frac{k n \hat{C}_{13}}{h \hat{C}_{33}} & \frac{k m \hat{C}_{23}}{h \hat{C}_{33}} & 0 & 0 & 0 \\ -k n \frac{\hat{C}_{13}}{\hat{C}_{33}} 2 & \alpha 1 & \alpha 2 & 0 & 0 & 0 \\ -k m \frac{\hat{C}_{23}}{\hat{C}_{33}} & \beta 1 & \beta 2 & 0 & 0 & 0\end{array}\right]$

avec

$$
\left\{\begin{array}{l}
\alpha 1=\frac{1}{\mu}\left(\rho s^{2} h^{2}+k m^{2} \hat{C}_{66}-\left(\frac{\hat{C}_{13}^{2}}{\hat{C}_{33}}-\hat{C}_{11}\right) k n^{2}\right) \\
\alpha 2=\frac{1}{\mu}\left(k n k m \hat{C}_{66}-\left(\frac{\hat{C}_{13} \hat{C}_{23}}{\hat{C}_{33}}-\hat{C}_{12}\right) k n^{2}\right)
\end{array}\right.
$$

$$
\begin{aligned}
& \left\{\begin{array}{l}
\beta 1=\frac{1}{\mu}\left(\hat{C}_{66}-\left(\frac{\hat{C}_{23} \hat{C}_{13}}{\hat{C}_{33}}-\hat{C}_{12}\right)\right) k n k m \\
\beta 2=\frac{1}{\mu}\left(\rho s^{2} h^{2}+k n^{2} \hat{C}_{66}^{2}-\left(\frac{\hat{C}_{23}^{2}}{\hat{C}_{33}}-\hat{C}_{22}\right) k m^{2}\right)
\end{array}\right. \\
& k n=n \pi \kappa \quad k m=m \pi \kappa \quad \kappa=h / l
\end{aligned}
$$

La solution générale de l'équation 5 est donnée par:

$$
\begin{aligned}
& \bar{\Psi}(\zeta, s)=\exp \left(\left(\zeta-\zeta_{k-1}\right) A_{n m}(s)\right) \bar{\Psi}\left(\zeta_{k-1}, s\right) \\
& \left(\zeta_{k-1} \leq \zeta \leq \zeta_{k} ; \quad k=1,2, . . N\right)
\end{aligned}
$$

avec $\zeta_{0}=0$ et $\zeta_{k}=z_{k} / h=\sum_{j=1}^{k} h_{j} / h$ et $h_{\mathrm{k}}$ est l'épaisseur de la $\mathrm{k}^{\text {ième }}$ couche.

Prenant par exemple $\zeta=\zeta_{\mathrm{k}}$ dans l'équation (7), on aura la relation entre les vecteurs d'état des surfaces supérieure et inférieure pour la $\mathrm{k}^{\mathrm{ième}}$ couche comme suit :

$\Psi_{1}^{(k)}(s)=M_{k}(s) \Psi_{0}^{(k)}(s)$

avec $M_{k}(s)=\exp \left(\left(\zeta_{k}-\zeta_{k-1}\right) A_{n m}(s)\right)$. 


\subsubsection{Modélisation de l'interface viscoélastique:}

On suppose que l'interface entre la $\mathrm{k}^{\mathrm{eme}}$ et la $(\mathrm{k}+1)^{\mathrm{eme}}$ couche est viscoélastique, et que :

$\sigma_{z}^{(k+1)}=\sigma_{z}^{(k)} \quad, \quad \tau_{x z}^{(k+1)}=\tau_{x z}^{(k)} \quad, \quad \tau_{y z}^{(k+1)}=\tau_{y z}^{(k)} \quad \grave{a} \quad z=z_{k}$ $u^{(k+1)}=u^{(k)}+\delta^{(k)} \quad, \quad v^{(k+1)}=v^{(k)}+\delta^{(k)} \quad, \quad W^{(k+1)}=W^{(k)}$

avec $\delta^{(k)}$ et $\delta^{(k)}$ sont les déplacements relatifs dans la $\mathrm{k}^{\text {ième }}$ interface dans les directions $\mathrm{x}$ et $\mathrm{y}$. Elles sont reliées aux contraintes de cisaillement par les relations [7] :

$\left(\begin{array}{l}\tau_{x z}^{(k)}=\eta_{0 x}^{(k)} \delta_{x}^{(k)}+\eta_{1 x}^{(k)} \dot{\delta}_{x}^{(k)} \\ \tau_{y z}^{(k)}=\eta_{0 y}^{(k)} \delta_{y}^{(k)}+\eta_{1 y}^{(k)} \dot{\delta}_{y}^{(k)}\end{array}\right) \quad \grave{a} \quad z=z_{k}$

Où le point exprime la dérivée par rapport au temps. On suppose que ces déplacements s'écrivent sous la forme :

$\delta^{(k)}=h \bar{\delta}^{(k)}(x, t) \cos (n \pi \xi) \sin (m \pi \eta)$

$\delta_{v}^{(k)}=h \bar{\delta}^{(k)}(y, t) \cos (n \pi \xi) \sin (m \pi \eta)$

Dans le domaine de Laplace, la relation (10) se ramène à:

$$
\left(\begin{array}{c}
\bar{\tau}_{x z}^{(k)}(\zeta, s)=\left(\eta_{0 x}^{(k)}+s^{*} \eta_{1 x}^{(k)}\right) \bar{\delta}_{x}^{(k)}(\zeta, s) \\
\bar{\tau}_{y z}^{(k)}(\zeta, s)=\left(\eta_{0 y}^{(k)}+s^{*} \eta_{1 y}^{(k)}\right) \bar{\delta}_{y}^{(k)}(\zeta, s)
\end{array}\right) \quad \grave{a} \quad z=z_{k}
$$

En tenant compte des équations (9) et (11), l'équation (8) associée à la $\mathrm{k}^{\text {ième }}$ couche est donnée par :

$$
\begin{aligned}
& \bar{\Psi}_{0}^{(k+1)}(\zeta, s)=\bar{\Psi}_{1}^{(k)}(\zeta, s)+\bar{Q}^{(k)}(\zeta, s) \\
& \text { avec } \bar{Q}^{(k)}=\left[0, \bar{\delta}_{x}^{(k)}, \bar{\delta}_{y}^{(k)}, 0,0,0\right]^{T} \\
& \bar{\Psi}_{1}^{(k+1)}(\zeta, s)=M_{k+1}(s) \bar{\Psi}_{1}^{(k)}(\zeta, s)+M_{k+1}(s) \bar{Q}^{(k)}(\zeta, s)
\end{aligned}
$$

La relation entre la couche $\mathrm{N}$ et la couche 0 est donnée par :

$$
\begin{aligned}
& \bar{\Psi}_{1}^{(N)}(\zeta, s)=T(\zeta, s) \bar{\Psi}_{0}^{(1)}(\zeta, s)+\bar{S} \\
& \text { avec } T(\zeta, s)=\prod_{j=N}^{1} M_{j}(\zeta, s), \bar{\Psi}_{0}(\zeta, s)=\bar{\Psi}\left(\zeta_{0}, s\right) \\
& \text { et } \bar{S}=M_{N} \bar{Q}^{(N-1)}+M_{N} M_{N-1} \bar{Q}^{(N-2)}+\cdots+\prod_{j=N}^{1} M_{j} \bar{Q}^{(1)}
\end{aligned}
$$

$\mathrm{S}$ est associé aux interfaces viscoélastiques et s'annule dans le cas d'un stratifié parfaitement collé.

A l'aide de ce formalisme mathématique on peut prédire les déplacements et les contraintes de la structure multicouche dans n'importe quelle coordonnée $\zeta$ de l'épaisseur.

Pour cela, il suffit de déterminer l'expression explicite du vecteur initial $\bar{\Psi}_{0}$.

\subsubsection{Prise en compte des conditions aux bords}

On suppose que la plaque est soumise à une force externe $\sigma_{z}$, appliquée sur la surface supérieure $\zeta=1$ de la plaque ure 1), sinusoïdale et de variations spatiales (9) traires:

$\sigma_{z}(\xi, \eta, \zeta=1, s)=p_{z}(\xi, \eta, s)=\sum_{n=0}^{\infty} \sum_{m=0}^{\infty} \mu \bar{P}_{z}(s) \sin (n \pi \xi) \sin (m \pi \eta)$

Avec

$\bar{P}_{z}(s)=\bar{\Sigma}_{z}(\zeta=1, s)=\frac{4}{\mu} \int_{0}^{1} \int_{0}^{1} p_{z}(\xi, \eta, s) \sin (n \pi \xi) \sin (m \pi \eta)$

On suppose aussi que la surface inférieure de la plaque est reposée sur une fondation viscoélastique de type Winkler-Pasternak (figure 1). La contrainte à $\zeta=0$ est donnée sous la forme :

$$
\begin{aligned}
& \sigma_{z}(\xi, \eta, \zeta=0)=s c \bar{W}_{0}+K_{w} \bar{W}_{0}-a^{-2} K_{p x} \frac{\partial^{2} \bar{W}_{0}}{\partial \xi^{2}}-b^{-2} K_{p y} \frac{\partial^{2} \bar{W}_{0}}{\partial \eta^{2}} \\
& \sigma_{z}(\xi, \eta, \zeta=0)=\beta(s) \bar{W}_{0}
\end{aligned}
$$

Où $\left(\mathrm{K}_{\mathrm{w}}=\mathrm{K}_{\mathrm{px}}=\mathrm{K}_{\mathrm{py}}\right)$ sont les paramètres de fondations viscoélastiques et $W_{0}(\xi, \eta)=W(\xi, \eta, \zeta=0)$ est le déplacement transversal à $\zeta=0$.

En utilisant (4); l'équation (15) se ramène à

$\bar{\Sigma}_{\zeta}(\zeta=0)=\beta(s) \bar{W}(\zeta=0)$ avec

$\beta(s)=1 / \mu\left(s c+K_{w} h+\frac{K_{p x}}{h} k n^{2}+\frac{K_{p y}}{h} k m^{2}\right)$

On suppose que les conditions aux bords restantes sont données par:

$$
\begin{array}{lll}
\Sigma_{\zeta h}=\bar{\Sigma}_{\zeta h} & ; & \Gamma_{1 h}=\bar{\Gamma}_{1 h} \\
\Gamma_{3 h}=\bar{\Gamma}_{3 h} & ; & \Gamma_{10}=\bar{\Gamma}_{10}
\end{array} \quad \Gamma_{30}=\hat{\Gamma}_{30}
$$

En tenant compte de ces conditions aux bords, on obtient :

$\left\{\begin{array}{l}\bar{U}(\zeta, s) \\ \bar{V}(\zeta, s) \\ \bar{W}(\zeta, s)\end{array}\right\}=\left[\begin{array}{lll}t_{12} & t_{13} & \beta t_{11}+t_{14} \\ t_{52} & t_{53} & \beta t_{51}+t_{54} \\ t_{62} & t_{63} & \beta t_{61}+t_{64}\end{array}\right]^{-1}\left(\left\{\begin{array}{l}\bar{\Sigma}_{\zeta h} \\ \bar{\Gamma}_{1 h} \\ \bar{\Gamma}_{3 h}\end{array}\right\}-\left[\begin{array}{ll}t_{15} & t_{16} \\ t_{55} & t_{56} \\ t_{65} & t_{66}\end{array}\right]\left\{\begin{array}{l}\bar{\Gamma}_{10} \\ \bar{\Gamma}_{30}\end{array}\right\}\right)$

En rajoutant à ce vecteur les expressions de $\Sigma_{\zeta 0}, \Gamma_{10}$ et $\Gamma_{30}$, on peut en déduire les contraintes et déplacements $\bar{\Psi}(\zeta, s)$ dans n'importe quelle coordonnée $\zeta$. Notons que ces résultats sont obtenus dans le domaine de Laplace. Pour les réponses temporelles, un algorithme d'inversion numérique de transformée de Laplace est nécessaire.

\subsubsection{Méthode d'inversion}

Il est impossible de trouver la transformation inverse de Laplace analytiquement pour des solutions aussi compliquées des déplacements et contraintes obtenues 
précédemment. La méthode d'inversion numérique est alors utilisée.

La formule d'inversion complexe de la transformée de Laplace s'écrit sous la forme [9]:

$\Psi(\zeta, t)=\frac{1}{2 \pi i} \int_{\alpha-i \infty}^{\alpha+i \infty} \bar{\Psi}(\zeta, s) e^{s t} d s$

avec $\mathrm{i}^{2}=-1$, et $\alpha$ est nombre réel. Dans ce travail, l'approche de Durbin [10] pour le calcul numérique de la transformée de Laplace dans un intervalle $\left[\begin{array}{ll}0 & \mathrm{~T}_{0}\end{array}\right]$ est utilisée pour la discrétisation de la forme complexe (19). La formulation suivante est utilisée:

$\Psi(\zeta, t)=\frac{2 e^{\alpha t}}{T_{0}}\left[\frac{1}{2} \operatorname{Re}\{\bar{\Psi}(\zeta, \alpha)\}+\sum_{i=1}^{N}\left(\begin{array}{l}\left\{\operatorname{Re}\left\{\bar{\Psi}\left(\zeta, \alpha+i \frac{2 \pi l}{T_{0}}\right)\right\}\right\} * \cos \left(\frac{2 \pi l}{T_{0}} t\right) \\ -\left\{\operatorname{Im}\left\{\bar{\Psi}\left(\zeta, \alpha+i \frac{2 \pi l}{T_{0}}\right)\right\}\right\} * \sin \left(\frac{2 \pi l}{T_{0}} t\right)\end{array}\right]\right)$

avec $\bar{\Psi}(\zeta, s)$ est le vecteur d'état et $\mathrm{N}$ la constante de la troncature et la valeur suggérée $d u$ « $\alpha \mathrm{T}_{0} »$ est comprise entre 5 et 10 .

La méthodologie, ainsi développée, permet d'obtenir le comportement tridimensionnel des plaques et poutres multicouches avec ou sans interfaces. Les réponses statiques et dynamiques des contraintes et déplacements selon l'épaisseur peuvent être analysées pour différents types de couches, d'interfaces et de charges.

\section{Résultats Numériques}

Considérons une plaque simplement appuyée constituée du matériau Graphite-Epoxy dans chaque couche. Les propriétés de ce matériau sont exprimées par les constantes d'élasticité suivantes :

Table 1. Constantes d'élasticité du matériau Graphite-Epoxy

\begin{tabular}{|l|l|l|l|l|l|}
\hline Csts & $(\mathrm{Gpa})$ & Csts & $(\mathrm{Gpa})$ & Csts & $(\mathrm{Gpa})$ \\
\hline C11 & 183.443 & $\mathrm{C} 44$ & 2.870 & $\mathrm{C} 23$ & 3.918 \\
\hline C22 & 11.662 & $\mathrm{C} 12$ & 4.363 & $\mathrm{C} 55$ & 7.170 \\
\hline C33 & 11.662 & $\mathrm{C} 13$ & 4.363 & $\mathrm{C} 66$ & 7.170 \\
\hline
\end{tabular}

En premier lieu, on suppose que la plaque est constituée de trois couches, que l'interface entre les couches est parfaite et que la configuration d'angle d'orthotropie est : $\left[0 \% / 90^{\circ} / 0^{\circ}\right]$.

Les contraintes $\sigma_{\mathrm{z}}, \tau_{\mathrm{yz}}$ et $\tau_{\mathrm{xz}}$, obtenues numériquement, sont données sur la figure 2 .

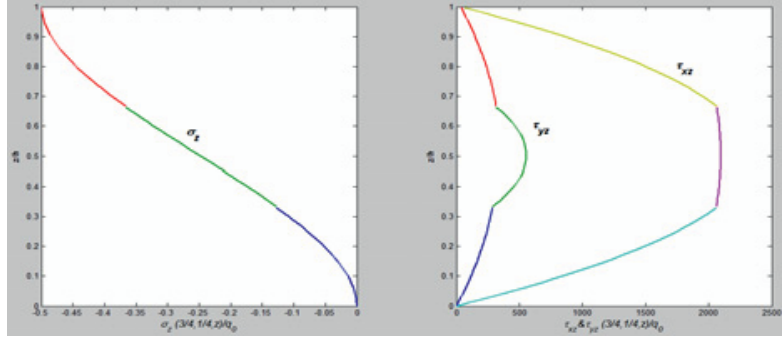

Fig. 2: $\sigma_{\mathrm{z}}, \tau_{\mathrm{yz}}$ et $\tau_{\mathrm{xz}}$ selon l'épaisseur d'une plaque laminée $\left[0^{\circ} / 90^{\circ} / 0^{\circ}\right]$

Les résultats présentés sur la figure 2 coïncident parfaitement avec ceux obtenus Ajdour et Azrar [11] en utilisant le modèle de Stroh.

En guise de généralisation, une structure laminée composée de cinq couches, avec une répartition d'angle arbitraire est considérée. Les variations de $\sigma_{\mathrm{z}}, \tau_{\mathrm{yz}}$ et $\tau_{\mathrm{xz}}$ selon l'épaisseur d'une plaque laminée, les contraintes sont données sur la figure 3 .

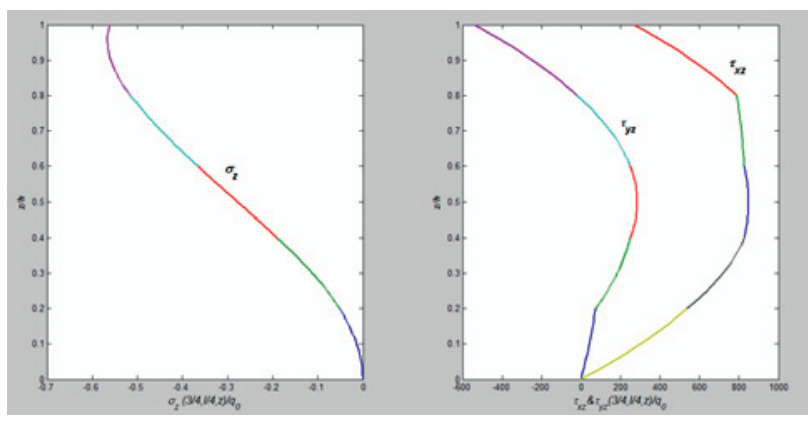

Fig. 3: Cinq couche : Plaque laminées $\left[0^{\circ} / 30^{\circ} / 60^{\circ} / 90^{\circ} / 45^{\circ}\right]$

Sur les figures 2 et 3, on peux voir clairement que l'angle d'orthotropie n'a pas d'effet sur la contrainte $\sigma_{z}$. En l'occurrence pour les autres contraintes $\tau_{\mathrm{yz}}$ et $\tau_{\mathrm{xz}}$ on constate une variation de leurs valeurs avec le changement de l'angle d'orthotropie.

On considère ensuite un stratifié constitué de 5 couches avec la configuration $\left[0 \%-15^{\circ} /-60^{\circ} / 0^{\circ} / 45^{\circ}\right]$ pour les angles d'orthotropie. On suppose que les interfaces une et trois sont viscoélastiques et les interfaces deux et quatre sont parfaites, avec les paramètres suivants $\eta_{0 x}{ }^{(1)}=\eta_{0 x}{ }^{(3)}$ et $\eta_{0 \mathrm{y}}{ }^{(1)}=2 * \eta_{0 \mathrm{y}}{ }^{(3)}$ et un rapport $\kappa=\mathrm{h} / \mathrm{l}=0.01$.

La figure 4 présente les contraintes $\sigma_{\mathrm{z}}, \tau_{\mathrm{yz}}$ et $\tau_{\mathrm{xz}}$ et les déplacements $\mathrm{u}, \mathrm{v}$ et $\mathrm{w}$ en fonction de temps pour la position $\xi=3 / 4, \eta=1 / 4$.

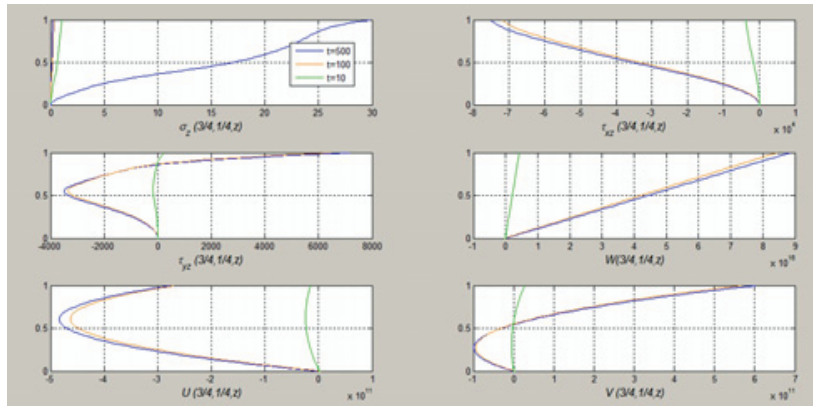

Fig. 4: Réponse temporelle pour plaque laminées $\left[0^{\circ} / /-15^{\circ} /-60^{\circ} / / 0^{\circ} / 45^{\circ}\right]$ 
Ces figures montrent l'évolution des contraintes, cisaillements et déplacements en fonction du temps pour l'épaisseur $\zeta=1$.

\section{Conclusion}

L'approche développée dans cet article permet de faire la modélisation et simulation numérique des comportements statique et dynamique des plaques multicouches à effets d'interfaces reposant sur une fondation viscoélastique de type Winkler et Pasternak. Les réponses statiques et dynamiques à interfaces visqueuses ont été obtenues. Un nombre arbitraire de couches ayant différents angles d'orthotropie peut être considéré à fin d'optimiser les contraintes et déplacements selon l'épaisseur. Cette approche méthodologique permet l'étude et le design des plaques multicouches ayant des contraintes et déplacements optimisés selon l'épaisseur. Les interfaces viscoélastiques, permettant la conception de plaques et poutres à comportement passif dont l'amortissement est amplifié, peuvent être considérées.

\section{Références}

1. Calme O. Suivi en fatigue et vieillissement humide de la ténacité d'interfaces de délaminage dans des assemblages multicouches comprenant des composites carbone-époxy à renforts textiles: Thèse de Doctorat, Université Claude Bernard Lyon I; (2007)

2. Yu HY, Wei YN, Chiang FP. Load transfer at imperfect interfaces-dislocation-like model. Int. J. Eng. Sci. (2002);40:1647-1662.

3. He LH, Jiang J. Transient mechanical response of laminated elastic strips with viscous interfaces in cylindrical bending. Compos. Sci. Technol. (2003);63:821-828.

4. Chen WQ, Lee KY. Time-dependent behaviors of angle-ply laminates with viscous interfaces in cylindrical bending. Eur. J. Mech. - Asolids. (2004);23:235-245.

5. Hashin Z. Composite materials with viscoelastic interphase: creep and relaxation. Mech. Mater. (1991);11:135-148.

6. Fan H, Wang GF. Interaction between a screw dislocation and viscoelastic interfaces. Int. J. Solids Struct. (2003);40:763-776.

7. Yan W, Chen W. Time-dependent response of laminated isotropic strips with viscoelastic interfaces. J. Zhejiang Univ. Sci. (2004);5:1318-1321.

8. Jones RM. Mechanics Of Composite Materials. 2 Sub. CRC Press; (1998).

9. Hildebrand FB. Advanced calculus for applications. Prentice-Hall; (1976).

10. F. Durbin. Numerical inversion of Laplace transforms: an effective improvement of Dubner and Abate's method. Comput J. (1973);17:371-6.
11. Ajdour M, Azrar L. Analytical Solutions of Loaded Multifunctional Multilayered Plates. Adv. Mater. Res. (2013);682:127-134. 